\title{
On Modeling Proto-Imitation in a Pre-associative Babel
}

\author{
Elpida Tzafestas \\ Institute of Communication and Computer Systems \\ National Technical University of Athens \\ Zographou Campus, 15773 Athens, Greece \\ brensham@softlab.ece.ntua.gr
}

\begin{abstract}
In this paper we present a model of generative proto-imitation that replicates external signals without associating with objects, as in higher-level imitation. A mixed population of adults, that have fixed associations objectssignals, and infants, that do not have associations but imitate unconditionally, endowed with a kinship and interaction structure, allows infants to develop signal affinity with their kin in a variety of conditions and within an initial random world, i.e. in a Babel. Our results indicate that the communicative value of imitation can be discovered after the basic apparatus is in place, rather than that communication is the end to which imitation is the means.
\end{abstract}

Keywords: Imitation, proto-imitation, kinship, Baldwin effect, development.

\section{Introduction}

Research in imitation spreads in various disciplines (for an overview see [1]) and generally centers around two major themes: the role of imitation in social interaction and communication and the mechanism by which imitated responses are produced. Functional studies related to social behavior and communication are common both in psychology and theoretical biology and rely on the implicit assumption that imitation is mainly a means to (learn to) communicate or interact socially [2][3][4]. Studies related to the neural mechanisms behind imitative response generation investigate mainly phenomena of neonatal or early infant imitation and are tackling questions such as the degree to which neonatal imitation is goal-directed, motivated and selective [5][6]. Other important issues we retained from the literature as specifications for modeling are:

- Imitation should start as a reactive or impulsive process and subsequently catalyze itself through the social interaction itself. This self-catalysis may be through direct training by adults; adult turn-taking in imitation is one such way of training [4].

- Imitation is a multi-level process found in many different forms of varying degrees of complexity in a vast number of animal species [7]. However, even animals that can imitate cannot learn to communicate at a 
human-like level of complexity and this is an instance of the major question in cognitive science "what makes humans different from animals" [8].

- When used in a communicative context, human-level imitation manages to co-exist with diverse, arbitrary sign systems, which is apparently contradictory with the effect of imitation [9][10].

- Disturbed versions of imitative mechanisms may be found in a spectrum of developmental disorders [11] and this is an indication that imitation cannot be studied outside a development context, at least functionally [12].

We are therefore developing a model that allows proto-imitation outside explicit communication but may allow emergence of communication in the medium or long term ontogenetically. This model separates response imitation from response association to external meaning so as to make room both for species that can imitate but not associate meaning and for disabled humans that cannot associate well or even proto-imitate well. We work at the response imitation level to show that a wealth of phenomena may later emerge and especially those related to direct associations.

\section{The Agent Model: Generative Imitation}

The usual agent model found in the literature (see for example [3]), supposes the existence of $\mathrm{M}$ objects with one signal associated with each object. The term "object" can denote anything from an individual or an inanimate object to an action or an event, in short any external thing that can be referred to. The signals are supposed to transfer information about the objects and can take values in any physical medium that an agent is able to use; however, the typical case is to think of signals as vocalizations. An imitative process is one that allows one agent to learn to use another agent's signal to refer to the same object. As such, imitation allows to two or more agents to communicate by using the same signals for the same objects. One common formalization of this imitation process [9] in a population of agents is through the use of a MxL language matrix per agent where each entry denotes the probability for the agent to refer to object $\mathrm{i}(\mathrm{i}=1,2, \ldots, \mathrm{M})$ by using the signal $\mathrm{j}(\mathrm{j}=1,2, \ldots, \mathrm{L})$. This setup presupposes that associations between objects and signals should exist for imitation to take place and that the role of imitation is to make the associations of different agents converge to one common language. This assumption is partly due to the adoption of the language domain as the experimental field of imitation par excellence.

Our own model of imitation of adult agent responses does not assume any prior association to any external object. Instead we model the way an infant agent recognizes and reproduces an observed signal coming from an adult agent and standing for the response to a perceived object without having access to the object itself and thus without associating with it. Our model is a functional model of a neural structure that generates responses to match and replicate an external stimulus, i.e. the signal received (a similar generative model has been reported in [13]). We opted for a study with the aid of a functional model of the neural structure rather than the structure itself, because we did not want to constrain our results within the possibilities of a given structure. We are rather seeking the organizational properties that such a structure should have to allow imitation. 
We begin therefore by adopting the view that the ontegenetic development at the neural level follows the same principles as Darwinian evolution at the population level ([14]) and that any novel responses should be generated internally and selected within the environment rather than be directly "taught" by it. By applying this view to the proto-imitation level of low-level reproduction of external signals, we get a model where continuous internal generation of responses combined with an environmental selection (i.e. reinforcement through actual response matching to external signal) allows various imitative phenomena within a population of agents. Association of signals to objects can appear later, if at all. We use real-valued signals as in [2] instead of the more common discrete or symbolic signals ([3][15] etc.), because we feel we should not ignore the continuous nature of real-world signals (especially vocalizations) and because we want to explicitly model the distance between agent responses.

An agent possesses a number of internal "frequencies" or eigenfrequencies (these could correspond to real vocal parameters or to neural patterns) that have varying degrees of affinity to a given signal: because frequencies as well as signals take real values in $[0,1]$, affinities of frequencies will also take real values in the same interval.

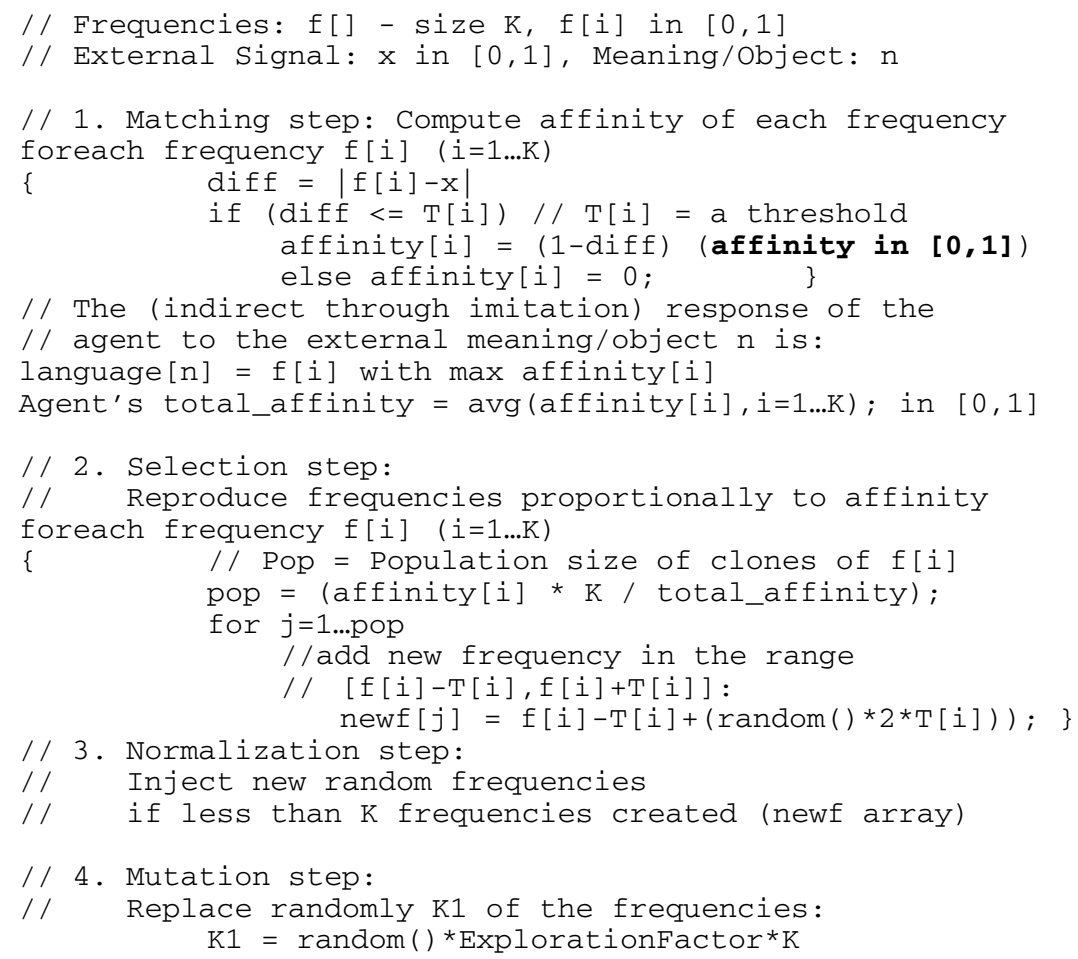

Fig. 1. The eigenfrequency-based functional model of generative imitation 
The response to a signal is the eigenfrequency with the highest affinity. At each step, new eigenfrequencies are generated proportionally to the affinity of the previous ones. The highest matching frequencies reproduce massively, while the lowest ones vanish and are replaced by newly generated random eigenfrequencies. An exploration factor is also defined, which is the maximum percentage of random eigenfrequency replacement independently of affinity. The overall affinity of an agent to an external signal is the average affinity of all its frequencies, thus it is internally generated and not externally imposed/designed in any way. This measure expresses how "well" an agent recognizes and can reproduce a signal and may therefore serve as a basis for subsequent emergence of communication. This model is summarized in Fig. 1.

\section{Experiments I: Adults, Infants and Kinship}

We have simulated populations of agents that follow the previous imitation model. In the same spirit as [3][15] we have separated the learning phase from the adult mode of behavior. Because in our system we have no predefined associations between objects and vocalizations we use two classes of agents, the adult and the infant agents. The former are supposed to have stabilized and to always respond to a particular object with one particular vocalization, while the latter are in a learning stage and are imitating the agents they encounter. Social encounters are based on the obvious intuition that infants are nurtured by a limited set of "parents" or kin agents and therefore they encounter most often or even exclusively these agents. All such parental relations are initialized randomly within the overall population with the aid of the kin factor parameter, which is the number of parents or kin agents per infant.

In each simulation cycle, every infant encounters an adult selected randomly from its kin population, unless social noise is present. Each encounter involves the adult agent acting as a sender of one or more signals and the infant agent acting as a receiver that imitates the received signals. This process uses two parameters, the number $\mathrm{M}$ of actual external objects refered to by the adult agents and the imitation factor which is the maximum number of signals received and imitated on every encounter (imitation factor $\leq \mathrm{M}$ ). All adult vocalizations as well as all infant frequencies are initialized randomly in the range [0,1]. This combination of uniformed reactive imitation with a tight social interaction structure allows infants to develop high affinity with their kin, where affinity with one or more agents is defined as the average affinity for all external objects. We remind however that an infant cannot associate objects and vocalizations and its current signal (word) for a particular object is the last emitted one, as shown in Fig. 1. Figures 2 and 3 show how average infant affinities develop in the case of a single or two parents, when refering to one or more objects. In all cases, the affinity with kin is clearly higher than the affinity with adults in general and it develops slower as the number of refering objects increases. Final kin affinity is also lower as the number of kin agents increases. It is relevant to note that while average kin affinity rises, average adult affinity remains at best the same, thus the affinity with non-kin agents is on average negatively affected by the buildup of affinity with kin. Note also that in our simulations we have chosen $\mathrm{K}$ (the number of frequencies) to be high (50), to induce high initial affinity and learning speed. 

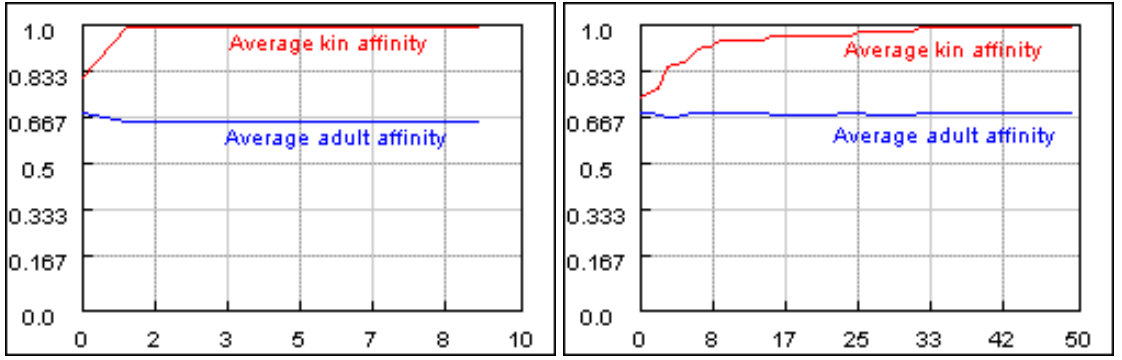

Fig. 2. ( $x=$ time, $y=$ affinity in $[0,1]) 10$ adults, 10 infants, random parental relations. (a) Kin factor $=1, M=1$, imitation factor $=1$ (single parent and object). (b) Kin factor $=1, M=3$, imitation factor $=2$ (single parent, many objects).
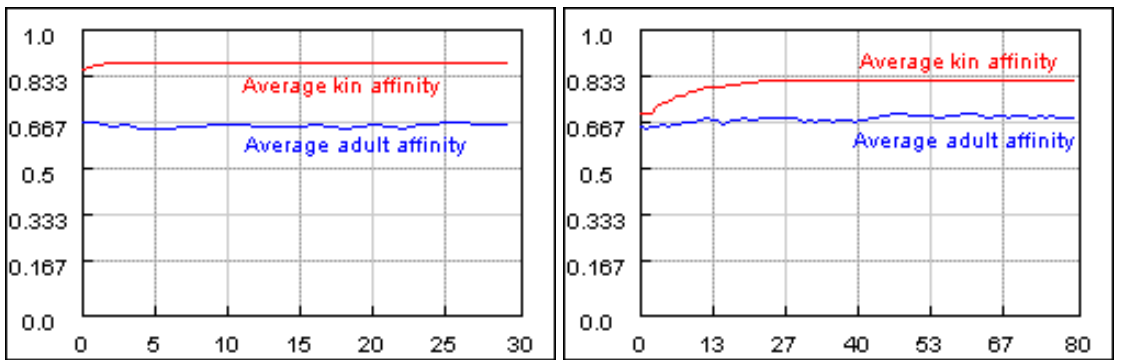

Fig. 3. ( $x=$ time, $y=$ affinity in $[0,1]) 10$ adults, 10 infants, random parental relations. (a) Kin factor $=2, M=1$, imitation factor $=1$ (many parents, single object). (b) Kin factor $=2, M=3$, imitation factor $=2$ (many parents, many objects).
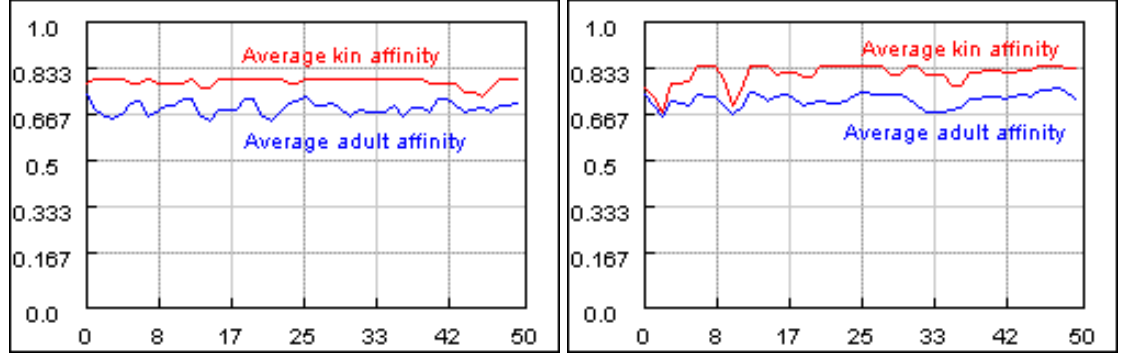

Fig. 4. ( $x=$ time, $y=$ affinity in $[0,1]) 10$ adults, 10 infants, random parental relations. Kin factor $=2, M=1$, imitation factor $=1$ (many parents). (a) Social exploration factor $=0.1$, interaction factor $=5$. (b) Signal noise probability: 0.1 .

In figure 4 we are introducing noise in the system either as social noise or signal noise. Social noise is implemented with the aid of a social exploration factor which is the probability that an infant will encounter a non-kin agent belonging to a larger group of predefined agents (the interaction factor is the total number of agents that form an infant's social environment, kin and non-kin). Signal noise is implemented as a probability with which the received signal from another agent will be mutated to a 
random value (again in the range $[0,1]$ ). Even when noise is present, the infants are still able to develop higher affinities with kin than with other agents.

In figure 5, we replicate the experiment of [3] as can be implemented in our system: we examine role model or fitness-based matching and random matching as social interaction rules in place of our kin-based rule. Fitness-based matching means that an adult is selected for interaction by an infant with a probability proportional to its communicative fitness, that is in our case its average affinity with the agents it interacts with. Although the system is expected to be self-catalyzed by its own behavior (accidental communicative success will lead to higher probability to interact again in the future), it does not seem to allow consistent development of higher affinity with interacting agents. This is obvious in figure $5 \mathrm{~b}$, where the affinities for one particular infant are depicted: average kin affinity fluctuates above and below the average adult affinity levels without being able to settle (notice that in this case, where no kinship is defined, what we measure as kin affinity is actually the affinity with the interacting agents). The results are similar in the case of random matching. The lesson that can be drawn from this experiment is that informed matching is unnecessary for development of affinity with other agents; rather a blind observational imitation mechanism is sufficient, as has been also pointed out by [15].

Our final experiment, whose results are given in fig. 6 , involves cultural learning from generation to generation. More specifically, we design a system where after 100 cycles of execution (recall that, as shown in previous figures, the populations stabilize in less than 30 cycles), the whole population is replaced by a new population where the adults are swept from the system, the infants become adults with no possibility to

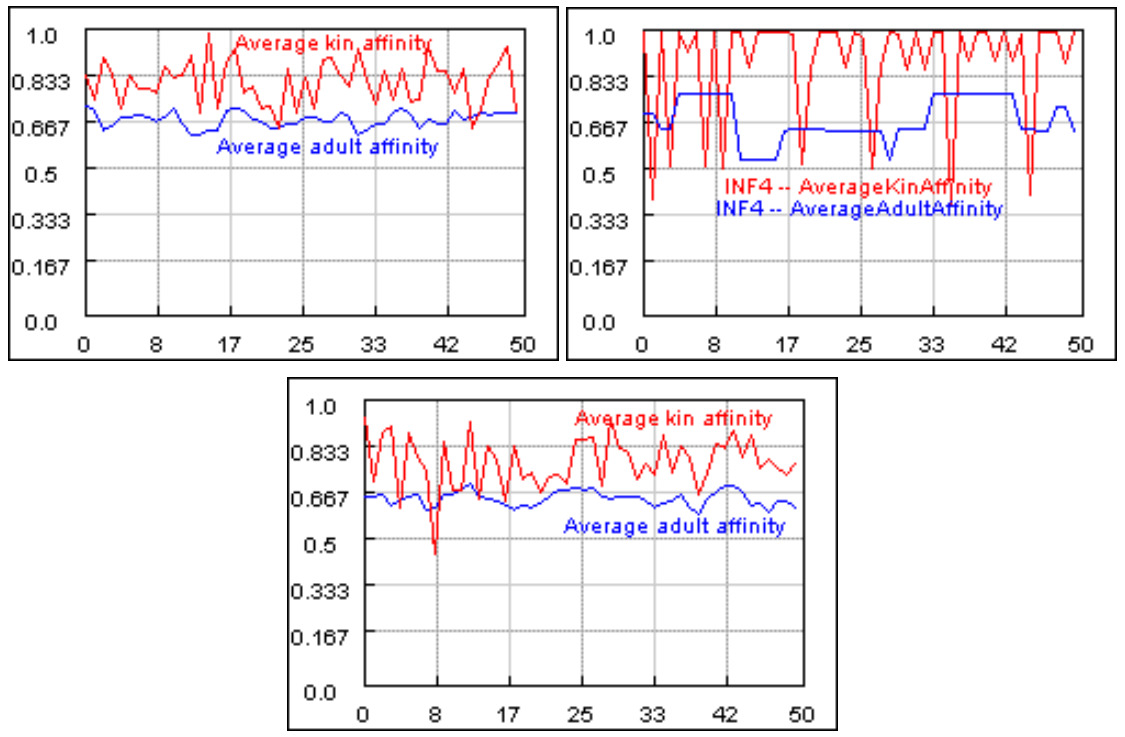

Fig. 5. ( $x=$ time, $y=$ affinity in $[0,1]) 10$ adults, 10 infants, $M=1$, imitation factor $=1$, no noise. (a) Fitness-based matching, average population measurements, (b) One typical infant's measurements in the previous experiment, (c) Random-based matching, average population measurements. 


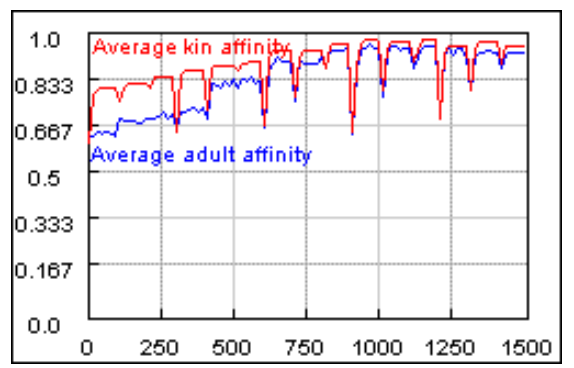

Fig. 6. $(x=$ generation, $y=$ affinity in $[0,1]) 10$ adults, 10 infants, kin factor $=2, M=5$, imitation factor $=3$, no noise. 15 generations of 100 cycles each.

imitate anymore and newly born, randomly initialized infants enter the population in the place of the former infants. We run this system for a number of generations and we find, not too unsurprisingly, that subsequent generations achieve much higher average adult and kin affinity scores, approaching the theoretical maximum of 1, even for big language sets $(M=5$, imitation factor=3 in the example). This is an example of how the Baldwin effect [16] can arise in the cultural domain: cultural instead of genetic inheritance combined with generation overlapping can play the role of the acquired features transmission mechanism, thus in the long term leading to a system that has learnt adaptive or even "optimal" behaviors. It also parallels the results of [17] who also found that language becomes highly regular if it is transmitted from generation to generation. Finally, this result further supports our initial hypothesis that the communicative function of an individualistic sign system can evolve after the structural apparatus for proto-imitation is in place: it is not difficult to imagine that an initial Babel population of agents with fully diverse vocalization sets endowed with such a rudimentary imitation mechanism can develop and "discover" a few generations later that signs are shared and can use them in a communicative manner, although the mechanics of such an extension will be most probably fairly complex.

\section{Experiments II: Social Structure and Individual Development}

Having completed the experiments of the previous section, we wondered how the otherwise obvious infant-adult attachment relations could be dissected in detail so as to identify exactly how social structure, age or developmental differences can influence the emergence of affinity or dynamic kinship in agent populations. In figure 7, we give the results of a single-parent system where one infant is reinitialized with a new parent before stabilization. Correspondingly, figure 8 gives the results of a singleparent system where one infant is reinitialized with a new parent after it has stabilized. In both cases, if the agent is given enough time it can find the new stable position of maximum affinity with the new parent. However, re-initialization slows down the learning process because the infant frequency system at reinitialization is not completely random but more or less tuned to the former target, thus re-learning is slower than initial learning. 

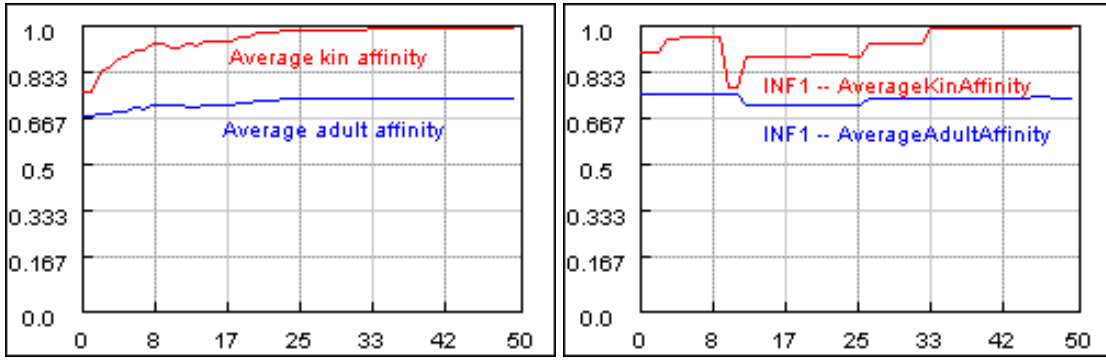

Fig. 7. $(x=$ time, $y=$ affinity in $[0,1]) 10$ adults, 10 infants, kin factor $=1, M=3$, imitation factor $=$ 2 , no noise. At $t=10$ reinitialization of an infant with a new parent. (a) Average population measurements, (b) The infant measurements.


Fig. 8. $(x=$ time, $y=$ affinity in $[0,1]) 10$ adults, 10 infants, kin factor $=1, M=3$, imitation factor $=$ 2 , no noise. At $\mathrm{t}=30$ reinitialization of an infant with a new parent. (a) Average population measurements, (b) The infant measurements.

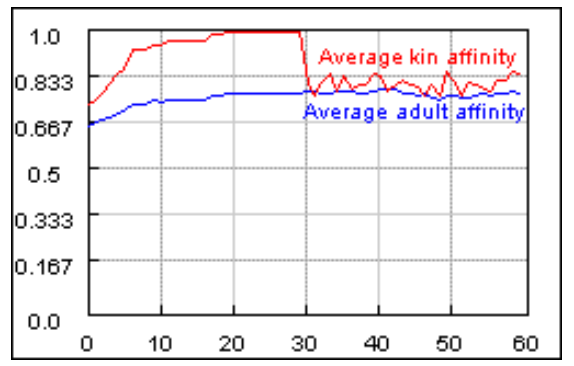

Fig. 9. $(x=$ time, $y=$ affinity in $[0,1]) 10$ adults, 10 infants, kin factor $=1, M=3$, imitation factor $=$ 2 , no noise. At $\mathrm{t}=30$ allow infants to match randomly and imitate all adults. Average adult affinity does not change. Compare the second part of the chart (after $t=30)$ with figure $5 \mathrm{c}$. After the system has stabilized, random matching and imitation does not seem to harm.

A final experiment concerning the passage to adult age consists in reverting to random matching and imitation when the system has stabilized. In this case, counterintuitively, the average adult affinity does not drop, but the average kin affinity drops and fluctuates a little above the adult level (fig. 9). This result combined with the 

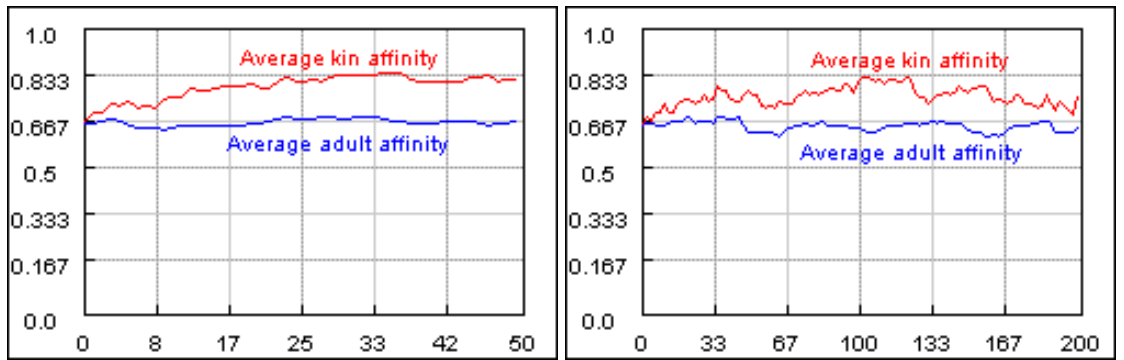

Fig. 10. ( $x=$ time, $y=$ affinity in $[0,1]) 10$ adults, 10 infants, $M=3$, imitation factor $=2$, no noise. (a) Every infant has one parent and one friend infant. (b) Every infant has two parents and as many brethren as defined by shared parental links.
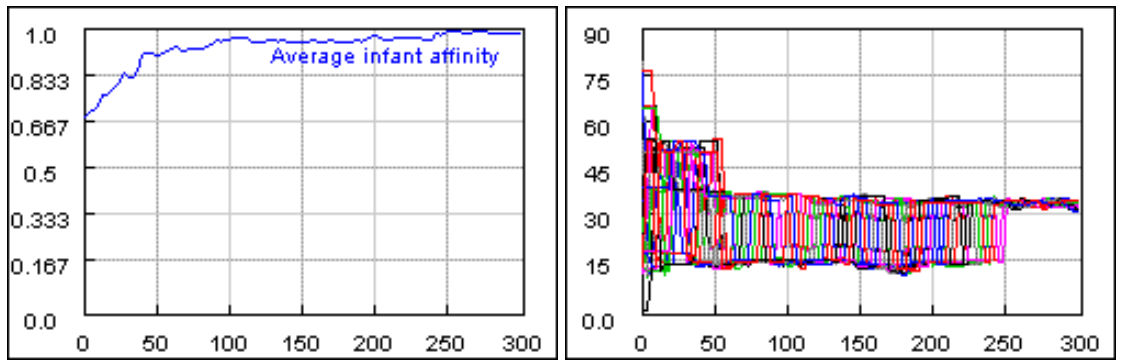

Fig. 11. No adults, 20 infants, $M=3$, imitation factor $=2$, no noise, random matching model. (a) ( $x=$ time, $y=$ affinity in $[0,1])$ Average population affinity, (b) ( $x=$ time, $y=$ signals in $[0,1] x 100)$ All 20 agents vocalizations for one of the objects. If given sufficient time, they converge to one common vocalization for all agents. Vocalizations are amplified for visualization purposes.

previous ones means that the proto-imitative system is very vulnerable during stabilization from a random starting point but fairly robust when stabilized.

Because in reality, a young agent does not only interact with adults and it is not logical to assume that even then it only imitates adults, we extend the kinship relations by including either random other infants (friends) or the brothers of the infant. In figure 10, we give the results of a pair of such experiments. In both cases, and despite inferior convergence to high kin affinity values, there is clear separation of the trends for average adult and average kin affinity.

Although the distinction between infants and adults is defined only with respect to their ability to imitate, it makes sense to investigate what would happen in the extreme case of an all-infant population with random interactions. As shown in fig. 11, the system develops very high average affinity close to the theoretical maximum of 1 . Interestingly, if given enough time such a system converges to a single shared language (and this is why final average affinity is so high). So, what can only evolve culturally if age-dependent or other imitation differences exist, can appear within one generation that is imitative and initially "tabula rasa". Once more this supports our hypothesis that proto-imitation precedes any notion of communication, it is purely reactive and does not need any association with meaning. Associations and the discovery of communicative value only come at a later stage. 


\section{Conclusion}

We have presented a model of generative proto-imitation of signals without association with an external meaning. Our model purported to show that an initial non-functional "Babel" of diversely vocalizing agents, if endowed with a nonassociative proto-imitation mechanism, allows communication to emerge at a subsequent, possibly associative, stage. We have shown how our model can yield a wealth of population phenomena that are generally taken with given explicit associations and especially how affinity can develop between initially tabula rasa imitative infants and adult agents to which infants are attached in a variety of ways. Other prominent results include the cultural development from generation to generation and convergence to one single "language", as well as an impressive onegeneration convergence in the case an all-infant population. Immediate future work underway includes both an extension of the model with an association component as well as experimentation with explicit agent development that is absent in the current version of the model.

\section{References}

1. Hurley, S., Chater, N.: Perspectives on imitation in animals and humans. MIT Press, Cambridge (2005)

2. Suzuki, J., Kaneko, K.: Imitation games. Physica D 75, 328-342 (1994)

3. Nowak, M.A., Plotkin, J.B., Krakauer, D.C.: The evolutionary language game. J. Theor.Biol. 200, 147-162 (1999)

4. Eckerman, C.O., Stein, M.R.: How imitation begets imitation and toddlers' generation of games. Devel. Psychol. 26, 370-378 (1990)

5. Gergely, G., Bekkering, H., Király, I.: Rational imitation in preverbal infants. Nature 415, 755 (2002)

6. Bekkering, H., Wohlschläger, A., Gattis, M.: Imitation of gestures in children is goaldirected. Quarterly J. of Exp. Psychol. 53A, 153-164 (2000)

7. Caldwell, C.A., Whiten, A.: Evolutionary perspectives on imitation: Is a comparative psychology of social learning possible? Anim. Cogn. 5, 192-208 (2002)

8. Oliphant, M.: Rethinking the language bottleneck: Why don't animals learn to communicate? In: Presented at the 2nd Intern. Conf. on the Evolution of Language (1988)

9. Krakauer, D.C.: Selective imitation for a private sign system. J. Theor. Biol. 213, 145-157 (2001)

10. Grassly, N.C., von Haeseler, A., Krakauer, D.C.: Error, population structure and the origin of diverse sign systems. J. Theor. Biol. 206, 369-378 (2000)

11. Stieglitz Ham, H., Corley, M., Rajendran, G., Carletta, J., Swanson, S.: Imitation of meaningless gestures in individuals with Asperger syndrome and high-functioning autism. J. Autism Dev. Disord. 38, 569-573 (2008)

12. Karmiloff-Smith, A.: Development itself is the key to understanding developmental disorders. Trends in Cognitive Sciences 2, 389-398 (1998)

13. Tzafestas, E.S.: Compromising algorithmicity and plasticity in autonomous agent control systems: The autonomous cell. J. Intell. Syst. 9, 135-176 (1999) 
14. Edelman, G.M.: Neural darwinism. Basic Books, New York (1987)

15. Oliphant, M.: The learning barrier: Moving from innate to learned systems of communication. Adaptive Behavior 7, 371-384 (1999)

16. Baldwin, J.M.: A new factor in evolution. Amer. Natur. 30, 441-451 \&536-553 (1896)

17. Vogt, P.: Cumulative cultural evolution: Can we ever learn more? In: Proceedings Simulation of Adaptive Behavior (2006) 